\title{
Participación en redes organizacionales y uso de las tecnologías de la información y la comunicación, un estudio de su impacto en los resultados de la cooperativas de enseñanza de la comunidad valenciana
}

\author{
Vanessa Campos Climent $^{1}$ y Cristina Navarro Babiera ${ }^{2}$
}

Recibido: 4 de enero de 2017 / Aceptado: 19 de marzo de 2017

Resumen. Las cooperativas de enseñanza o escuelas cooperativas forman parte de uno de los sectores más desconocidos dentro del sistema educativo valenciano. Estas cooperativas van más allá de suponer una mera realidad de empresas prósperas, aparecen en el panorama educativo como un modelo organizacional alternativo de la enseñanza con una clara vocación transformadora. Inmersas en otras concepciones de la educación dentro del propio sistema, las cooperativas de enseñanza plantean una oferta educativa sensiblemente diferente a la que podemos considerar tradicional, siendo generadoras de valor económico y social. Por este motivo, puede resultar relevante determinar los factores que influyen sobre los resultados económicos y sociales de estas organizaciones.

Así, el objetivo del presente estudio es analizar los efectos de dos herramientas de apoyo en la gestión como son: el uso de las tecnologías de la información y la comunicación (TICs) y la participación en redes organizacionales sobre los resultados tanto económicos como sociales de las cooperativas de enseñanza. En base a un estudio empírico sobre una muestra de 23 cooperativas de enseñanza de la Comunidad Valenciana, esta investigación muestra la existencia de una relación significativa entre el uso de las TICs y los resultados económicos de las cooperativas de enseñanza valencianas. Mientras que no fue posible establecer una relación significativa entre el uso de las TICs y el resultado social de las mismas. Asimismo, tampoco se pudo establecer la existencia de una relación significativa entre la participación de las cooperativas de enseñanza valencianas en redes organizacionales y sus resultados tanto económicos como sociales.

Palabras clave: Empresas sociales; eficacia económica; eficacia social; TICs; alianzas; stakeholders.

Claves Econlit: L200; M150; M210; O330; P130.

[en] Participation in organizational networks and use of information and comunication technologies. An study about their impact on performance of comunidad valenciana co-operative schools

\footnotetext{
1 Universitat de València, España

Dirección de correo electrónico: vanessa.campos@uv.es

2 Universitat de València, España

Dirección de correo electrónico: cristinanb12@gmail.com
}

Abstract. Co-operative schools belong to one of the most unknown realities within the education system. These co-operatives go beyond to be a merely profitable entrepreneurial reality, appearing as an alternative educational model with clear transformational purposes. Co-operative schools are 
immerses in the educational system but propose a different educational offer from those other approaches that we can consider the traditional ones. For these reasons, it can be interesting to identify the factors that have an impact on the economic and social performance of such organizations.

The current study purpose is to analyze the impact of the employ of ICTs and the participation in organizational networks, as support tools for co-operative schools management, on both economic and social performance. To this end we perform an empirical study based on a sample of 23 Valencian co-operative schools. Results show the existence of a significant positive impact of ITCs employment on economic performance, while it has not been possible to establish the existence of any kind of significant impact of ICTs on social performance. Research neither allows to identify any significant relationship between the participation in organizational networks and the co-operative schools performance, both economic and social performance.

Keywords: Social enterprises; economic effectiveness; social effectiveness; ICTs; alliances; stakeholders.

Sumario. 1. Introducción. 2. Uso de las TICs y participación en redes organizacionales, impacto en los resultados. 3. Metodología. 4. Análisis de resultados. 5. Conclusiones. 6. Referencias bibliográficas.

Cómo citar: Campos, V. y Navarro, C. (2017) Participación en redes organizacionales y uso de las tecnologías de la información y la comunicación, un estudio de su impacto en los resultados de la cooperativas de enseñanza de la comunidad valenciana. REVESCO. Revista de Estudios Cooperativos, $\mathrm{N}^{\circ} 124$, pp. 32-46. DOI: 10.5209/REVE.56130.

\section{Introducción}

Las cooperativas de enseñanza forman parte de un modelo, hasta ahora poco investigado, que ofrece una oferta diferenciadora que sobrepasa la dicotomía de escuela pública-privada (Fuente et al., 2002). Habitualmente cuando se habla de centros educativos, se habla de centros públicos y privados, y en este último caso de los centros concertados, que son mayoría dentro del sector privado (Fernández y Muñiz, 2012). Uno de los sectores más desconocidos dentro de los centros concertados es el colectivo de escuelas cooperativas o cooperativas de enseñanza, un modelo de empresa insólito que poco a poco está tomando más fuerza y visibilidad, el cual cuenta con una larga trayectoria histórica.

La realidad es que en la Comunidad Valenciana el modelo de escuelas cooperativas ha sufrido históricamente una marginación institucional. Actualmente, las cooperativas de enseñanza presentan un buen posicionamiento en el sector en un momento en que el sector está atravesando una crisis que más que profesional es social, también estructural y, por descontado, económica. La ideología cooperativista así como, las estructuras organizativas que se han desarrollado en los últimos años han conseguido que este modelo comporte una diferenciación clara sobre el resto de centros docentes tradicionales. Por tanto, esta diferenciación les otorga una ventaja competitiva que las sitúa en mejor posición para afrontar su futuro en entornos turbulentos (Inglada et al., 2015).

Las cooperativas de enseñanza se enfrentan al siglo XXI con nuevos planteamientos globales que afectan a la empresa y a la educación en general (Fuente et al., 2002). Por una parte, las tecnologías de la información y la comunicación han supuesto una profunda transformación de la naturaleza del 
trabajo (Fernández-Guadaño, 2000) potenciando nuevos modelos de enseñanza y también la competitividad dentro del sector. Por otra parte, la creación y participación de redes organizacionales ha pasado a ser determinante para mantener y extender la sociedad cooperativa.

Dados estos dos planteamientos, el presente trabajo tiene como objetivo determinar la contribución de herramientas de apoyo a la gestión como el uso de las TICs y la participación en redes organizacionales sobre los resultados económicos y sociales de las cooperativas de enseñanza. A través de técnicas estadísticas como son el análisis de componentes principales y la regresión múltiple procederemos a testar si los factores antes señalados, influyen significativamente en los resultados tanto económicos como sociales de las cooperativas de enseñanza.

Para alcanzar los objetivos planteados, en la siguiente sección se caracteriza a las cooperativas de enseñanza valencianas (CEV) para identificar y posicionar este modelo organizacional dentro del sistema educativo. Además, se examina el uso de las TICs y la participación en redes organizacionales analizando la situación actual y evaluando su papel dentro de la gestión de las CEV. En la tercera sección, se presenta la metodología empleada, en la cuarta se recogen los principales resultados y finalmente, en la quinta y última sección se plantean las conclusiones del estudio, sus limitaciones y las futuras líneas de investigación.

\section{Uso de las TICs y participación en redes organizacionales, impacto en los resultados}

Diversos estudios han coincidido en señalar el papel que juegan las TICs así como la gestión de dichas tecnologías en la mejora de los resultados de las organizaciones empresariales (De Haes y Van Grembergen, 2009; Prasad et al., 2012; Wu et al., 2015). Si bien estudios que centren su análisis empírico en el impacto de las TICs en organizaciones de la economía social son todavía escasos. Entre ellos, encontramos el estudio de Gargallo y Pérez (2009) que establece una relación positiva entre TICs y productividad en una muestra de 20 empresas manufactureras que operan bajo la forma jurídica de cooperativa de trabajo asociado. Por otra parte, Sanchis et al. (2015) señalan el uso de las TICs como uno de los factores que contribuyen al éxito de las cooperativas de nueva creación. Sin embargo, no existen estudios empíricos que relacionen el uso de las TICs y los resultados organizativos en el sector de las cooperativas de enseñanza.

El uso de las TICs está siendo un reto decisivo en el mundo de la educación, los avances que ofrecen son idóneos para facilitar la participación de los distintos grupos de personas que intervienen en el proceso educativo. Actualmente, estas tecnologías están en evolución constante y acelerada, tanto en número como en complejidad. Diversos países desarrollados del mundo ya han incorporado las TICs a la educación, sumergiéndose en la globalización y la mundialización del conocimiento; es decir, creando un espacio global de conocimiento. Vivimos en un mundo dominado por la tecnología y su uso está generando nuevas y diferentes formas de desarrollo (Sánchez, 2002). Dentro de este contexto, la educación se encuentra en un período de redefinición y renovación a todos los niveles, buscando 
la transformación y adecuación del proceso de enseñanza-aprendizaje a la realidad antes mencionada (Sefton-Green, 2006). Las TICs son consideradas una herramienta poderosa para el cambio y la reforma educativa, diversos estudios han demostrado que un uso adecuado de las TICs puede elevar la calidad educativa $(\mathrm{Fu}$, 2013). Además, esta herramienta es capaz de involucrar a todos los grupos de interés (stakeholders) en la cooperativa posibilitando la efectiva obtención de sus objetivos, favoreciendo su relación y agilizando los procesos de gestión mediante nuevos canales de tratamiento y acceso a la información.

Existen una serie de elementos que pueden facilitar o dificultar el éxito del uso de las TICs en el escenario de las cooperativas de enseñanza. Desde la década 2000, se han venido desarrollando estudios que los exploran como por ejemplo, los trabajos de Cuban (2001) y Tearle y Golder (2008), los cuales agrupan esos elementos en tres dimensiones: (1) el clima escolar, que hace referencia a la visión compartida existente alrededor del aprovechamiento de las TICs; (2) la gestión de las TICs o el presupuesto, planes de implementación, apoyo técnico, formación y estrategias de apoyo puestas en marcha; y por último, (3) el conocimiento de las TICs, entendido como la capacidad de los centros de enseñanza para garantizar la adquisición y actualización de equipos así como, la integración pedagógica, modificación de los contenidos y el desarrollo de métodos de evaluación adaptados (Gee y Hayes, 2011).

Otro factor a considerar es la participación en redes organizacionales. En la literatura es posible encontrar trabajos recientes que relacionan la participación en redes organizacionales con una mejora en los resultados de las empresas (Jenssen y Nybakk, 2013; Mc Adam et. al., 2016).

En el sector cooperativo, este factor proviene de la aplicación del sexto principio cooperativo: la cooperación entre cooperativas. La Declaración de Identidad Cooperativa realizada por la Alianza Cooperativa Internacional (ACI) en septiembre de 1995. Según García-Gutiérrez (2000), este principio permite consolidar y proyectar el ámbito empresarial y social de la cooperativa en entornos más amplios, mediante la colaboración práctica y rigurosa de otras cooperativas y otros niveles de cooperación. La cooperación entre cooperativas puede desarrollar eficazmente la competitividad del cooperativismo como sector y de las cooperativas como empresas, mediante redes cooperativas regionales, nacionales e internacionales.

Este principio surgió en buena medida como una respuesta frente a las demandas del sector que veía como aumentaba la dimensión media del resto de organizaciones con las cuales, las propias cooperativas competían. En efecto, viene a determinar un proceso en muchos casos de auténtica supervivencia (FernándezGuadaño, 2002).

Para la aplicación de este principio, existe la posibilidad de constituir diversas formas jurídicas como pueden ser las cooperativas de segundo grado, asociaciones, uniones y federaciones entre otras. Todas estas fórmulas favorecen el mejor cumplimiento del objeto social y la búsqueda de sinergias para la defensa de los intereses comunes bajo la consideración de que a través de la unión se consigue la fuerza para competir en los mercados a la vez que se consiguen determinados objetivos sociales. A pesar de tener diversas modalidades para su implementación, se podría señalar que la cooperación entre cooperativas es uno de los principios 
básicos del cooperativismo que más dificultades encuentra en el momento de su implantación en el mundo real.

Por otra parte, dentro del sector del cooperativismo de enseñanza, la VII Declaración Educativa propugnada por "La Unió de Cooperatives d'Ensenyament Valencianes" UCEV (2016) señala que las cooperativas de enseñanza pueden conseguir una mayor dimensión, obtener financiación, compartir dificultades, y en suma, competir mejor, no entre ellas mismas, si no frente al resto de centros docentes. Así, serán capaces de maximizar su impacto al colaborar las unas con las otras y buscar los beneficios de operar la organización a gran escala.

En definitiva, la finalidad es emprender un camino para hacer frente a mayores retos, la globalización, la competitividad, la sostenibilidad, el deterioro del medio ambiente y la crisis (Martínez, 2012).

En el caso particular de la Comunidad Valenciana, las cooperativas de enseñanza están agrupadas en redes organizacionales en forma de cooperativas de segundo grado y uniones como muestra la tabla 1.

Tabla. 1. Redes organizacionales de las Cooperativas de Enseñanza valencianas (2015)

\begin{tabular}{|c|c|}
\hline UeCoE & Unión Española de Cooperativas de Enseñanza \\
\hline UCEV & Unió de Cooperatives d'Ensenyament Valencianes \\
\hline AKOE & Cooperativa de segundo grado \\
\hline SITE & Cooperativa de segundo grado \\
\hline
\end{tabular}

Fuente: elaboración propia a partir de lados de la UCEV.

Así, el marco conceptual en el que se basa nuestra investigación contempla dos herramientas que pueden ser poderosas para el desarrollo de las cooperativas de enseñanza como son: el uso de las TICs y la cooperación entre cooperativas a través de la participación en redes organizacionales. Dos factores que dada su relevancia parecen indicar que condicionan la mejora y el progreso empresarial desde un punto de vista amplio de estas entidades. Es decir, pueden tener una influencia positiva sobre los resultados tanto económicos como sociales de las cooperativas de enseñanza.

Por tanto, proponemos testar las hipótesis siguientes:

H1: El uso de las TICs y la participación en redes organizacionales están positivamente relacionadas con mejores resultados económicos en las cooperativas de enseñanza.

H2: El uso de las TICs y la participación en redes organizacionales están positivamente relacionadas con mejores resultados sociales en las cooperativas de enseñanza.

Dichas hipótesis quedan modelizadas en las ecuaciones siguientes:

1. $\mathrm{RE}=\alpha 1+\beta 1.1 * \mathrm{UTIC}+\beta 1.2 * \mathrm{RORG}$

2. $\mathrm{RS}=\alpha 2+\beta 2.1 * \mathrm{UTIC}+\beta 2.2 * \mathrm{RORG}$ 
El modelo teórico enunciado en dichas hipótesis queda resumido en la figura 1.

Figura. 1. Modelo teórico propuesto

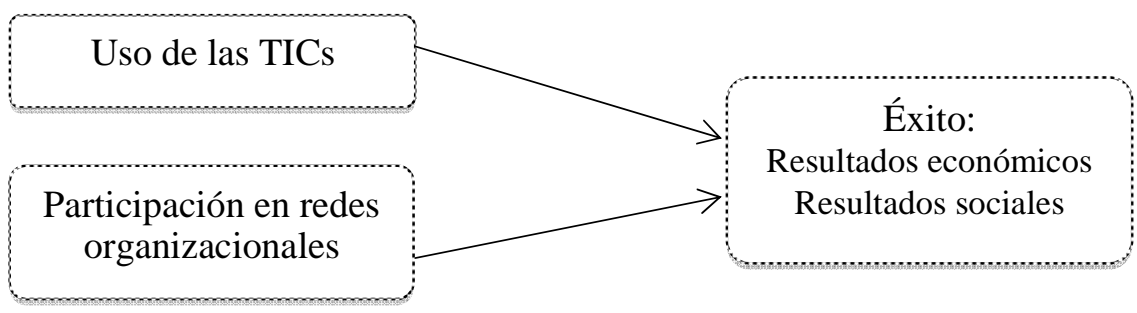

Fuente: elaboración propia.

\section{Metodología}

El presente estudio empírico se ha llevado a cabo sobre una muestra de 23 cooperativas de enseñanza de la Comunidad Valenciana. La población comprendía un total de 66 cooperativas a 30 de septiembre de 2016, por lo que la muestra analizada supone el $34,85 \%$ de las cooperativas de enseñanza valencianas. Para ello, se procedió al diseño de un cuestionario el cual fue validado por 3 personas que desarrollan su trabajo en áreas relacionadas con la gestión de una cooperativa de enseñanza valenciana. Durante el primer semestre de 2016 se envió el cuestionario a los centros mediante correo electrónico y además se hizo un seguimiento telefónico de las respuestas.

Para dar respuesta a las hipótesis formuladas, procedimos a determinar si herramientas de apoyo a la gestión como el uso de las TICs y la participación en redes organizacionales son capaces de influir de manera positiva y significativa sobre los resultados, tanto económicos como sociales, de las cooperativas de enseñanza.

Dado que el objetivo del estudio era determinar la existencia o no de relaciones positivas y significativas entre el uso de las TICs y la participación en redes organizacionales y los resultados (económicos y sociales), se optó por el uso de una metodología cuantitativa en dos etapas:

1. Se procedió a la agrupación de componentes a través del análisis de componentes principales (ACP). El ACP comporta la agrupación de variables que se correlacionan con más fuerza entre ellas (Pérez y Medrano, 2010).

2. Se procedió a testar mediante análisis de regresión múltiple la influencia de las variables independientes sobre las variables dependientes del modelo. La regresión múltiple permite determinar el comportamiento de las variables dependientes del modelo a través del comportamiento de las variables independientes del mismo (Levin y Rubin, 2004).

El tratamiento de datos se realizó mediante el paquete estadístico SPSS22. 


\section{Variables dependientes: Resultados económicos y resultados sociales}

Las empresas objeto del presente estudio, las cooperativas de enseñanza, son empresas que tal y como señalan Toledo-López et al. (2012) son empresas que persiguen objetivos sociales pero al mismo tiempo requieren ser rentables desde el punto de vista económico. Por ello, planteamos dos tipos de resultados: los resultados económicos y los resultados sociales.

El constructo resultados económicos (RE) de las cooperativas de enseñanza se ha medido a través de tres ítems: la mediana del retorno cooperativo obtenido en los últimos cuatro ejercicios económicos (RETCOR), ayudas financieras recibidas del sector público en los últimos cuatro ejercicios (AFIN), tomando AFIN el valor 2 si se han recibido ayudas y 1 en caso contrario, y la mediana de ayudas financieras del sector público recibidas en los últimos cuatro ejercicios económicos (MFIN). Para la definición de estos ítems se han adaptado las medidas propuestas por Sanchis et al. (2013).

El ítem RETCOR se ha medido mediante una escala de Likert 1-5, tomando el valor 5 si la mediana del retorno cooperativo de los últimos 4 ejercicios se encuentra muy por encima del sector, 4 ligeramente por encima del sector, 3 al mismo nivel del sector, 2 un poco por debajo del sector y 1 muy por debajo del sector. El ítem AFIN se ha medido como una variable dicotómica que ha tomado el valor 1 si la cooperativa no ha recibido ayudas financieras y 2 en caso de haberlas recibido. Finalmente, el ítem MFIN se ha medido mediante una escala de Likert 15 , tomando el valor 5 si la mediana de las ayudas financieras recibidas durante los últimos 4 ejercicios se encuentra muy por encima del sector, 4 ligeramente por encima del sector, 3 al mismo nivel del sector, 2 un poco por debajo del sector y 1 muy por debajo del sector o si no ha recibido ningún importe en concepto de ayudas financieras del sector público.

El constructo resultados sociales (RS) de las cooperativas de enseñanza se ha medido a través de cuatro ítems: el grado de satisfacción de los padres, madres o tutores (SAPA), la relación entre profesorado y alumnado (RPROAL), el porcentaje de profesorado que son socios-trabajadores de la cooperativa de enseñanza (SOCTRAB) y el grado de satisfacción del alumnado (SATAL). Para la definición de estos ítems se han adaptado las medidas propuestas por Sanchis et al. (2013) y Mc Guire et al. (2012).

El ítem SAPA se ha medido mediante una escala de Likert 1-5, siendo 5 completamente satisfecho, 4 bastante satisfecho, 3 indiferente, 2 poco satisfecho y 1 nada satisfecho. El ítem RPROAL se ha medido mediante una escala de Likert 15, siendo 5 excelente, 4 buena, 3 indiferente, 2 mala y 1 pésima o muy mala. El ítem SOCTRE se ha medido mediante una escala de Likert 1-5, tomando el valor 1 si en el último ejercicio menos del $60 \%$ del profesorado eran socios-trabajadores, 2 si están entre el 60\% y el 69\%, 3 si están entre el 70\%-79\%, 4 si están entre el $80 \%-89 \%$ y 5 si el porcentaje de socios-trabajadores supone el $90 \%$ o más del total del profesorado. Finalmente, el ítem SATAL se ha medido mediante una escala de Likert 1-5, siendo 5 completamente satisfecho, 4 bastante satisfecho, 3 indiferente, 2 poco satisfecho y 1 nada satisfecho. 


\section{Variables independientes: uso de las tecnologías de la comunicación y la información y participación en redes organizacionales}

El constructo uso de las tecnologías de la comunicación y la información (UTIC) se ha medido mediante siete ítems: uso de intranet (INTRA), uso del aprendizaje en línea (ALIN), frecuencia de uso del correo electrónico (FCOR), frecuencia en el uso de dispositivos digitales como ordenadores y/o tabletas (FOR), la frecuencia del uso de recursos digitales (FDIG), intensidad de la presencia de la cooperativa en redes sociales (NSOC) y la frecuencia en la actualización de la página web de la cooperativa (FWEB). Todos estos indicadores se midieron mediante una escala de Likert 1-5, tomando el valor 1 si la cooperativa se encontraba muy por debajo de la media del sector en su uso, 2 un poco por debajo de la media, 3 en la media, 4 un poco por encima de la media y 5 muy por encima de la media.

El ítem INTRA se ha medido como una variable dicotómica que toma el valor 1 en el caso que la cooperativa de enseñanza no disponga de intranet y 2 en el caso de disponer de ella. El ítem ALIN también se ha medido como una variable dicotómica que toma el valor 1 en el caso que la cooperativa de enseñanza no utilice el aprendizaje en línea y 2 en el caso de utilizarlo. El ítem FCOR se ha medido mediante una escala de Likert 1-5 siendo 1 si nunca se usa el correo electrónico y 5 si siempre se usa el correo electrónico como medio de comunicación. Por su parte el ítem FOR se ha medido empleando una escala de Likert 1-5, tomando el valor 1 si no se usan dispositivos digitales y 5 si siempre se usan dichos dispositivos en las tareas habituales. El ítem FDIG se ha medido mediante una escala de Likert 1-5, tomando el valor 1 si nunca se usan recursos digitales y 5 si el uso de recursos digitales es mucho más intenso que en el resto del sector. Por su parte los ítems NSOC y FWEB miden la presencia en redes sociales de la cooperativa y le frecuencia con la que se actualiza la web corporativa respectivamente, comparando la situación en la cooperativa en relación al resto del sector. Dichos ítems se midieron empleando una escala de Likert 1-5.

Finalmente, el constructo participación en redes organizacionales (RORG) se midió mediante cuatro ítems: la participación por parte de la cooperativa de enseñanza en algún tipo de red organizacional (XARCO), el número de redes organizacionales en las que participa la cooperativa (PXARCO), la frecuencia con la que la cooperativa realiza algún tipo de actividad relacionada con la red (FXARCO), el tipo de actividades que la cooperativa realiza a través de la red (AXARCO).

El ítem XARCO se ha medido como una variable dicotómica que toma el valor 1 si la cooperativa no participa en ningún tipo de red organizacional y 2 en caso de participar. El ítem PXARCO toma el valor del número de redes en las que participa la cooperativa. El ítem FXARCO se midió mediante una escala de Likert 1-5, tomando el valor 1 si la frecuencia con la que la cooperativa participa en las actividades de la red es muy baja, 2 baja, 3 en la media del sector, 4 alta y 5 si es muy alta. Por su parte el ítem AXARCO se ha medido como una variable cualitativa que clasifica en cinco grupos el tipo de actividades de la red en las que participa la cooperativa: siendo 1 actividades relacionadas con la innovación y metodología docente, 2 actividades relacionadas con la innovación tecnológica, 3 actividades relacionadas con la formación docente, 4 actividades relacionadas con 
el apoyo al cooperativismo y 5 actividades relacionadas con el desarrollo de herramientas facilitadoras del acceso a la financiación.

En la tabla 2 quedan recogidos de manera sintética los constructos definidos anteriormente, así como los ítems que se han utilizado en el presente estudio como instrumento de medida.

Tabla. 2. Variables del estudio

\begin{tabular}{ccc}
\hline Constructos & Ítems & Tipo de medida \\
\hline & INTRA & Dicotómica \\
Uso de las tecnologías de la & ALIN & \\
\cline { 2 - 3 } comunicación y la información & FDIG & \\
UTIC & FCOR & Escala Likert \\
& FOR & $1-5$ \\
Participación en redes & NSOC & \\
organizacionales & FWEB & Dicotómica \\
\cline { 2 - 3 } RORG & XARCO & Valor absoluto \\
\cline { 2 - 3 } & PXARCO & Escala Likert \\
\cline { 2 - 3 } Resultados económicos & FXARCO & Cualitativa \\
RE & AXARCO & Dicotómica \\
\hline \multirow{2}{*}{ Resultados sociales } & AFIN & Escala de Likert \\
RS & MFIN & $1-5$ \\
\hline & RETCOR & Escala de Likert \\
& SAPA & $1-5$ \\
\hline
\end{tabular}

Fuente: elaboración propia

\section{Resultados}

A continuación se analizan los resultados obtenidos del estudio realizado. Primeramente, respecto al análisis de componentes principales se ha seguido el procedimiento descrito por a Bartholomew et al. (2011) y Tabachnick et al. (2001).

La tabla 3 muestra la matriz de correlaciones de los ítems incluidos en el estudio, se ha optado por calcular el Rho de Spearman dado que la mayoría de los ítems han sido definidos como variables que toman valores discretos al ser de naturaleza dicotómica y ordinal según los casos.

Como puede observarse en la tabla 3, existen un gran número de correlaciones parciales entre ítems superiores al valor absoluto 0,3 que además son estadísticamente significativas, especialmente entre los ítems que están asociados al mismo constructo (tabla 2). Por lo que los datos, en principio, parecían apropiados para aplicar el ACP. 
Tabla. 3. Matriz de correlaciones, Rho de Spearman.

\begin{tabular}{|c|c|c|c|c|c|c|c|c|c|c|c|c|c|c|c|c|c|c|c|c|c|c|}
\hline & $7 F^{2}$ & & & & & & & & & & & PXARC & FXARC & AXARC & RETC & & & & & RPRO & SOCT & SATSO \\
\hline FWEB & $\frac{1.00}{1.000}$ & COK & $\mathrm{FCOR}$ & INTRA & FDIG & $\mathrm{NOR}$ & FOR & ALIN & $\mathrm{PSOC}$ & $\mathrm{NSOC}$ & XARCO & & 0 & & & AFIN & MFN & SATAL & SATPA & & & \\
\hline COR & $\frac{1,000}{0,403}$ & & & & & & & & & & & & & & & & & & & & & \\
\hline FCOR & 0,236 & 0,000 & 1.000 & & & & & & & & & & & & & & & & & & & \\
\hline NTRA & $-0,324$ & 0.233 & $\begin{array}{l}.578^{\prime \prime} \\
\end{array}$ & & & & & & & & & & & & & & & & & & & \\
\hline FDIG & 0,010 & 0.271 & $.715^{\prime \prime}$ & $-523^{\circ}$ & 1,000 & & & & & & & & & & & & & & & & & \\
\hline SOR & $.521^{\circ}$ & $.611 "$ & 0.349 & $-0,166$ & 0,217 & 1,000 & & & & & & & & & & & & & & & & \\
\hline FOR & 0.250 & 0.245 & $.585^{\prime \prime}$ & $-0,269$ & $.502^{\circ}$ & 0,405 & 1,000 & & & & & & & & & & & & & & & \\
\hline ALIN & $-0,227$ & $-0,363$ & . $750 "$ & 0,376 & $.471^{\circ}$ & $.464^{\circ}$ & . $4552^{\circ}$ & 1,000 & & & & & & & & & & & & & & \\
\hline PSOC & $-0,344$ & 0,117 & 0.369 & 0,402 & $-0,299$ & $-0,334$ & $-0,380$ & 0,239 & 1,000 & & & & & & & & & & & & & \\
\hline $\mathrm{NSOC}$ & $468^{\circ}$ & 0,095 & 0.295 & $.453^{\circ}$ & 0,073 & 0,216 & 0,313 & $-0,314$ & $-0,376$ & 1,000 & & & & & & & & & & & & \\
\hline XARCO & $-0,063$ & $-0,280$ & . $441^{\circ}$ & $.482^{\circ}$ & $.523^{\circ}$ & $-0,420$ & $-0,050$ & 0,376 & 0,402 & $-0,107$ & 1,000 & & & & & & & & & & & \\
\hline PXARCO & 0,022 & 0,163 & 0.345 & $-0,361$ & $.570^{\circ \prime}$ & 0,143 & 0,178 & $-0,281$ & $-0,301$ & 0,081 & $-748^{\prime \prime}$ & 1,000 & & & & & & & & & & \\
\hline FXARCO & 0,249 & 0,092 & $.507^{\circ}$ & - $491^{\circ}$ & $.423^{\circ}$ & 0,224 & $-0,007$ & $-0,233$ & $-0,324$ & 0,090 & " & $.693^{\prime \prime}$ & 1,000 & & & & & & & & & \\
\hline AXARCO & 0,315 & 0,376 & 0,394 & $-0,315$ & 0.293 & $.430^{\circ}$ & 0,054 & $-0,420$ & $-0,299$ & 0,114 & . $744^{\prime \prime}$ & $6511^{\prime \prime}$ & $.802^{\prime \prime}$ & 1,000 & & & & & & & & \\
\hline RETCOR & 0.260 & 0.208 & $6522^{\prime \prime}$ & $-452^{\circ}$ & 0,337 & 0,308 & $477^{\circ}$ & - $632 "$ & $-0,182$ & $.511^{\circ}$ & $-0,199$ & 0,199 & 0,320 & 0.211 & 1,000 & & & & & & & \\
\hline AFI & $-0,212$ & $-0,304$ & $.742^{\prime \prime}$ & $.499^{\circ}$ & . $\$ 01^{\circ}$ & $.442^{\circ}$ & $-0,159$ & $.650^{\circ 1}$ & 0,199 & $-0,262$ & $.495^{\circ}$ & $-0,215$ & $-0,391$ & $-0,272$ & $.6177^{\prime \prime}$ & 1,000 & & & & & & \\
\hline MFI & 0,422 & 0,369 & $.472^{\circ}$ & $-0,223$ & 0,226 & $.729^{\prime \prime}$ & 0,273 & $.488^{\circ}$ & $-0,251$ & $.424^{\circ}$ & $.490^{\circ}$ & 0,185 & 0,349 & 0.366 & $.563^{\prime \prime}$ & $.698 "$ & 1,000 & & & & & \\
\hline SATAL & . $5311 "$ & $.733 "$ & $-0,263$ & 0.334 & $-0,193$ & $-.737^{\prime \prime}$ & $-0,340$ & 0,384 & 0,306 & $-0,333$ & 0,334 & $-0,228$ & $-0,261$ & $-457^{\circ}$ & $-0,376$ & 0.281 & $-447^{\circ}$ & 1,000 & & & & \\
\hline SATPA & $.704^{\prime \prime}$ & $.452^{\circ}$ & 0,067 & 0,019 & 0,235 & . $6000 "$ & $-0,053$ & 0,033 & 0.255 & $-0,413$ & 0,224 & $-0,001$ & $-0,179$ & -0,383 & $-0,132$ & 0,074 & $4493^{\circ}$ & .681" & 1,000 & & & \\
\hline RPROAL & $.4499^{\circ}$ & $.501^{*}$ & $-0,257$ & 0.224 & $-0,083$ & . $568^{\prime \prime}$ & , $607 "$ & 0,376 & 0,402 & $-0,400$ & 0.224 & $-0,141$ & $-0,054$ & $-0,289$ & $-0,326$ & 0,059 & $\cdot 0,337$ & $.760^{\prime \prime}$ & .633" & 1,000 & & \\
\hline SOCTRP & 0,012 & . $477^{\circ}$ & 0.396 & $-0,343$ & 0,101 & 0,106 & 0,164 & -615" & 0,301 & 0,067 & $-0,062$ & $-0,011$ & 0,053 & 0,176 & $491^{\circ}$ & $-0,363$ & 0.209 & $-0,272$ & 0,131 & $-0,194$ & 1,000 & \\
\hline SATSOC & $-0,328$ & $-0,257$ & $-0,309$ & 0,198 & $-431^{*}$ & $-444^{\circ}$ & $-0,265$ & $-0,079$ & $.446^{\circ}$ & 0,131 & $432^{\circ}$ & $-0,228$ & $-437^{\circ}$ & $-0,242$ & $-0,090$ & 0.295 & $-0,333$ & 0,323 & 0,291 & 0,175 & 0,296 & 1,00 \\
\hline
\end{tabular}

Fuente: elaboración propia

Con el fin de valorar de manera más precisa la adecuación del ACP se calcularon el test de esfericidad de Barlett y el test de Kaiser-Meyer-Olin (KMO test). La tabla 4 recoge los resultados de dichos test.

Tabla. 4. Test de Barlett y KMO

\begin{tabular}{llcccc}
\hline & & UTIC & RORG & RE & RS \\
\hline KMO test & & 0,767 & 0,802 & 0,723 & 0,522 \\
\hline Bartlett test & $\begin{array}{l}\text { Aprox. Chi- } \\
\text { cuadrado }\end{array}$ & 64,190 & 71,612 & 24,071 & 40,340 \\
\cline { 2 - 6 } & gl & 21 & 6 & 3 & 6 \\
\cline { 2 - 6 } & Sig. & 0,000 & 0,000 & 0,000 & 0,000 \\
\hline
\end{tabular}

Fuente: elaboración propia

Como puede observarse en la tabla 4, los valores del KMO test están en todos los casos por encima del umbral mínimo del 0,5 y en la mayoría de los casos por encima del valor deseable del 0,7. Además, en todos los casos el test de Barlett resultó estadísticamente significativo al 0,01 . Por lo que concluimos que es aplicable el ACP para reducir el número de dimensiones de los constructos.

La tabla 5 recoge el resultado de aplicar el ACP usando la rotación Varimax. 
Tabla. 5. Resultados ACP

\begin{tabular}{cccc|c}
\hline Constructo & $\begin{array}{c}\text { Número de } \\
\text { factores } \\
\text { extraídos }\end{array}$ & Autovalor & $\begin{array}{c}\text { \% de varianza } \\
\text { extraída }\end{array}$ \\
\hline UTIC & 1 & 3,755 & 53,646 \\
\hline RORG & 1 & 3,276 & 81,897 \\
RE & 1 & 2,288 & 76,256 \\
\hline RS & 1 & 2,327 & 58,172 \\
\hline
\end{tabular}

Fuente: elaboración propia

A la vista de los resultados recogidos en la tabla 5, se conservó un factor por constructo. Se conservaron aquellos factores que presentaban autovalores mayores que uno y cuyo porcentaje de varianza extraída superaba el $50 \%$. Como se puede observar en la tabla 5, la varianza extraída por los factores se encuentra cercana al $60 \%$ o en la mayoría de los casos se acerca o supera el $80 \%$.

Seguidamente procedimos a la estimación de las dos ecuaciones del modelo planteado en la sección 2 del presente estudio mediante regresión lineal múltiple aplicando mínimos cuadrados ordinarios, para ello seguimos el procedimiento propuesto por Bartholomew et al. (2008). En la estimación de dichas ecuaciones se utilizaron como variables los factores extraídos a través del análisis de componentes principales. La tabla 6 recoge los resultados de la regresión lineal múltiple.

Tabla. 6. Resultados regresión lineal múltiple

\begin{tabular}{|c|c|c|c|c|}
\hline & \multicolumn{2}{|c|}{ RE } & \multicolumn{2}{|c|}{$\mathbf{R S}$} \\
\hline & Valor & Sig. & Valor & Sig. \\
\hline Constante & 0,08656 & 1,000 & $-0,0827$ & 1,000 \\
\hline UTIC & $0,652 * *$ & 0,003 & $-0,458 *$ & 0,063 \\
\hline RORG & 0,058 & 0,767 & $-0,044$ & 0,851 \\
\hline $\mathrm{R}^{2}$ & 0,467 & & 0,233 & \\
\hline $\mathrm{R}^{2}$ corregida & 0,410 & & 0,152 & \\
\hline $\begin{array}{l}\text { Durbin- } \\
\text { Whatson }\end{array}$ & 2,020 & & 2,295 & \\
\hline $\begin{array}{c}\text { ANOVA } \\
(\mathrm{F})\end{array}$ & $8,307 * *$ & 0,003 & $2,878^{*}$ & 0,081 \\
\hline
\end{tabular}

A la vista de los resultados recogidos en la tabla 6, el modelo estimado mediante regresión lineal múltiple es el siguiente:

1. $\mathrm{RE}=0,08656+0,652 * \mathrm{UTIC}+0,058 * \mathrm{RORG}$ 


\section{2. $\mathrm{RS}=-0,08278-0,458 * \mathrm{UTIC}-0,044 * \mathrm{RORG}$}

Como puede observarse en la tabla 6 , la $\mathrm{R}^{2}$ corregida de la ecuación 1 toma un valor de 0,410 . Lo que significa que el uso de las TICs y la participación en redes organizacionales explican conjuntamente el $41 \%$ del resultado económico de las cooperativas de enseñanza incluidas en la muestra. Además, el test ANOVA de la ecuación 1 resultó estadísticamente significativo al 0,05 , lo que nos indica que la ecuación es estadísticamente significativa y por tanto es posible explicar de manera fiable los resultados económicos de las cooperativas de enseñanza en base al uso que éstas hacen de las TICs y de su participación en redes organizacionales.

Respecto a la interpretación de los $\beta$ estimados de la ecuación 1 , tal y como recoge la tabla 6, el uso de las TICs tiene un impacto positivo y estadísticamente significativo al 0,05 en los resultados económicos. Así mismo, el impacto de la participación en redes organizacionales sobre los resultados económicos de las cooperativas de enseñanza es muy pequeño y además no resulta estadísticamente significativo.

Por su parte, la $\mathrm{R}^{2}$ corregida de la ecuación 2 toma un valor de 0,152 . Por lo que sólo el $15,2 \%$ de los resultados sociales de las cooperativas de enseñanza de la muestra pueden ser explicados mediante el uso de las TICs y la participación en redes organizacionales de dichas cooperativas. Además, el test ANOVA de la ecuación 2 resultó estadísticamente significativo al 0,1 por lo que el nivel de fiabilidad de la ecuación 2 para explicar los resultados sociales es menor que el de la ecuación 1 para explicar los resultados económicos.

Respecto a la interpretación de los $\beta$ estimados de la ecuación 2, se observa que ambos presentan signos negativos. Por lo que indicarían que tanto el uso de las TICs como la participación en redes organizacionales tendrían un impacto negativo en los resultados sociales de las cooperativas de enseñanza. Si bien, cabe señalar que solamente el $\beta$ estimado del uso de las TICs resultó estadísticamente significativo al 0,1 presentando un grado de confianza relativamente bajo y por debajo del umbral comúnmente aceptado del 0,05, mientras que el $\beta$ estimado para la participación en redes organizacionales no resultó estadísticamente significativo.

La hipótesis 1 del presente estudio proponía el uso de las TICs y la participación en redes organizacionales como factores que están positivamente relacionados con mejores resultados económicos en las cooperativas de enseñanza. De los $\beta$ estimados de la ecuación 1 se deduce que se puede constatar la existencia de una relación positiva y estadísticamente significativa al 0,05 entre el uso de las TICs y los resultados económicos de las cooperativas de enseñanza de la muestra. Mientras que para el caso de la participación en redes organizacionales y su impacto en los resultados económicos, dicha relación no ha resultado estadísticamente significativa al 0,05. Por lo que sólo es posible aceptar la hipótesis 1 parcialmente.

Por su parte, la hipótesis 2 proponía el uso de les TICs y la participación en redes organizacionales como factores que están positivamente relacionados con mejores resultados sociales en las cooperativas de enseñanza. Los resultados ponen de manifiesto un nivel bajo de la $\mathrm{R}^{2}$ corregida para la ecuación 2. Además los $\beta$ estimados para dicha ecuación presentan valores negativos, aunque sólo el $\beta$ 
estimado de la variable UTIC resultó estadísticamente significativo al 0,1. Por ello debemos rechazar la hipótesis 2.

\section{Conclusiones}

El presente estudio se plantea como objetivo establecer si ciertas herramientas de apoyo a la gestión, como las TICs y la participación en redes organizacionales, tienen un impacto positivo en los resultados organizativos de las cooperativas de enseñanza. Para ello, se ha procedido a considerar dos tipos de resultados organizativos: los resultados económicos y los resultados sociales de las cooperativas de enseñanza. En este sentido cabe señalar que mientras que la medición de los resultados económicos es una variable cuya medición está bastante establecida, no ocurre lo mismo con la variable resultados sociales. El presente estudio avanza en la medición de los resultados sociales a través del análisis factorial realizado, pues incluye en la medición de dichos resultados el output de la cooperativa de enseñanza de cara a los principales stakeholders de la misma completando la propuesta de Pedrosa (2009).

Por lo que respecta al uso de las tecnologías de la información y la comunicación, pocos estudios habían centrado su análisis en las empresas de la economía social. En este sentido, los resultados del presente estudio estableciendo un impacto positivo del uso de las TICs en los resultados económicos de las cooperativas de enseñanza apuntan en la misma dirección que el estudio de Gargallo y Pérez (2009), ya que aunque éste último relaciona positivamente TICs y productividad actualmente entre el mundo de academia y el mundo de la empresa hay acuerdo en considerar la productividad como un antecedente de los resultados económicos. Si bien, el presente estudio amplia los resultados a un sector mucho más intensivo en capital humano como lo es el de las cooperativas de enseñanza, este resultado puede estar apuntando a la importancia del uso de las TICs para la explotación de activos intangibles generadores de valor. Sin embargo nuestro estudio no nos ha permitido establecer la existencia de una relación positiva y significativa entre el uso de las TICs y los resultados sociales de las cooperativas de enseñanza.

Por otra parte no hemos podido establecer la existencia de una relación positiva y significativa entre la participación en redes organizacionales y los resultados organizativos de las cooperativas de enseñanza, dichas redes de cooperación se encuentran presentes y muy extendidas en el sector cooperativo en aplicación del principio de intercooperación cooperativa. Este resultado podría estar indicando que las cooperativas de enseñanza de la muestra, aunque participan mayoritariamente en redes, no están siendo capaces de trasladar los beneficios de la cooperación a los resultados (ni económicos ni sociales). Lo cual podría ser debido a la necesidad de desarrollar capacidades estratégicas entre los equipos directivos que les permitan capturar y explotar a nivel de cada cooperativa los beneficios que puede generar la cooperación en red.

Por tanto, las principales aportaciones del trabajo consisten en señalar el uso de las TICs como un antecedente de los resultados económicos de las cooperativas de enseñanza y en avanzar en el desarrollo de instrumentos de medida para medir el 
resultado social en empresas de la economía social. Así, las conclusiones del estudio pueden ser de interés tanto para los equipos directivos de las cooperativas de enseñanza y el sector cooperativo en general como para los actores políticos.

El presente estudio también presenta algunas limitaciones, como lo es el hecho de que la muestra de cooperativas de enseñanza se circunscriba a la Comunidad Valenciana. Por ello los resultados del mismo deben ser tomados con cautela. Como futuras líneas de investigación, las autoras están trabajando en ampliar la muestra a todo el territorio español.

\section{Referencias bibliográficas}

Bartholomew, D. J., Knott, M. y Moustaki, I. (2011) Latent variable models and factor analysis: A unified approach. John Wiley \& Sons.

Bartholomew, D. J., Steele, F., Galbraith, J. y Moustaki, I. (2008) Analysis of multivariate social science data. CRC press.

Cuban, L. (2001) Oversold and underused: Reforming schools through technology, 19802000. Cambridge: Oxford University Press.

De Haes, S. y Van Grembergen, W. (2009) An exploratory study into IT governance implementations and its impact on business IT alignment. Information Systems Management, Vol. 26, $\mathrm{N}^{\mathrm{o}}$ 2, pp 123-137.

Fernández-Guadaño, J. (2000) La realidad actual de las sociedades cooperativas en la educación. REVESCO. Revista de Estudios Cooperativos, № 71, pp. 55-76.

Fernández-Guadaño, J. (2002) La concentración cooperativa en el ámbito educativo español. Cayapa. Revista Venezolana de Economía Social, No 2, Vol. 4, pp. 69-82.

Fernández, R. y Muñiz, M. (2012) Colegios concertados y selección de escuela en España: un círculo vicioso. Presupuesto y gasto público, $\mathrm{N}^{\mathrm{o}} 67$, pp. 97-118.

Fu, J. S. (2013) ICT in education: A critical literature review and its implications. International Journal of Education and Development using Information and Communication Technology, № 9, Vol. 1, pp. 112-125.

Fuente, A. M., Delgado, M. y Álvarez, E. (2002) Las cooperativas de enseñanza como tercera vía dentro de nuestro sistema educativo: las cooperativas de trabajo asociado. Percepciones de sus directivos. Salamanca: Ediciones Universidad de Salamanca.

García-Gutiérrez Fernández, C. (2000) La intercooperación de las sociedades cooperativas en la actividad de la educación. REVESCO. Revista de Estudios Cooperativos, $\mathrm{N}^{\circ} 71$, pp. 173- 195.

Gargallo, A. y Pérez, F. (2009) El papel de las tecnologías de la información y la comunicación en las empresas de economía social. REVESCO. Revista de Estudios Cooperativos, $\mathrm{N}^{\mathrm{o}}$ 97, pp. 90-116.

Gee, J. P. y Hayes, E. R. (2011) Language and learning in the digital age. $1^{\text {a }}$ edición. New York: Routledge.

Inglada-Galiana, E., Sastre-Centeno, J. M. y Villarroya-Lequericaonandia, B. (2015) El cooperativismo en la educación. REVESCO. Revista de Estudios Cooperativos, $\mathrm{N}^{\mathrm{o}} 118$, pp. 122-147. DOI: 10.5209/rev_REVE.2015.n118.49059.

Jenssen, J. y Nybakk, E. (2013) Inter-organizational networks and innovation in small, knowledge-intensive firms: A literature review. International Journal of innovation management, $\mathrm{N}^{\mathrm{o}}$ 17, Vol. 2, pp.1-27. DOI: 10.1142/S1363919613500084.

Levin, R. y Rubin, D. (2004) Estadística para administración y economía. $7^{\mathrm{a}}$ edición. México: Pearson Educación. 
Martínez, A. (2012) Sobre el principio de cooperación entre cooperativas en la actualidad. Boletín de la Asociación Internacional de Derecho Cooperativo, No 46, pp. 133-146.

Mc Adam, M., Mc Adam, R., Dunn, A. y Mc Call, C. (2016) Regional Horizontal Networks within the SME Agri-Food Sector: An Innovation and Social Network Perspective. Regional Studies, $\quad \mathrm{N}^{\mathrm{o}}$ 50(8), pp. 1316-1329. http://dx.doi.org/10.1142/S1363919613500084.

Mc Guire, J.; Dow, S. y Ibrahim, B. (2012) All in the family? Social performance and corporate governance in the family firm. Journal of Business Research, Vol. 65, $\mathrm{N}^{\mathrm{o}} 11$, pp. 1643-1650.

Pedrosa, C. (2009) Modelos teóricos que nos ayudan a comprender el gobierno de las sociedades cooperativas, una apuesta por el enfoque de los stakeholders. Gestión joven, $\mathrm{N}^{\mathrm{o}} 4$, pp. 5-23.

Pérez, E. y Medrano, L. (2010) Análisis factorial exploratorio: bases conceptuales y metodológicas. RACC, Revista Argentina de Ciencias del Comportamiento, $\mathrm{N}^{\mathrm{o}} 2$, Vol. 1, pp. 58-66.

Prasad, A., Green, P. y Heales, J. (2012) On IT governance structures and their effectiveness in collaborative organizational structures. International Journal of Accounting Information Systems, № 13, Vol. 3, pp. 199-220.

Sánchez, J. (2002) Integración curricular de las TICs: conceptos e ideas. Santiago: Publicaciones Universidad de Santiago de Chile.

Sanchis, J. R., Campos, V. y Mohedano, A. (2013) Management in social enterprises: the influence of the use of strategic tools in business performance. International Entrepreneurship and Management Journal, $\mathrm{N}^{\circ}$ 9, Vol. 4, pp. 541-555.

Sanchis-Palacio, J. R., Campos-Climent, V. y Mohedano-Suanes, A. (2015) Factores clave en la creación y desarrollo de cooperativas. Estudio empírico aplicado a la Comunidad Valenciana. REVESCO. Revista de Estudios Cooperativos, $\mathrm{N}^{\circ} 119$, Tercer Cuatrimestre, pp. 183-207. DOI: 10.5209/rev_REVE.2015.n119.49153.

Sefton-Green, J. (2006) Youth, technology, and media cultures. Review of Research in Education, No 30 , pp. 279-306.

Tabachnick, B.; Fidell, L. y Osterlind, S. (2001) Using multivariate statistics. $5^{\text {a }}$ edición. New York: Pearson.

Tearle, P. y Golder, G. (2008) The use of ICT in the teaching and learning of physical education in compulsory education: how do we prepare the workforce of the future? European Journal of Teacher Education, № 31, Vol. 1, pp. 55-72.

Toledo-López, A., Díaz-Pichardo, R., Jiménez-Castañeda, J.C. y Sánchez-Medina, P. S. (2012) Defining success in subsistence business. Journal of Business Research, Vol. 65, $\mathrm{N}^{\mathrm{o}} 12$, pp 1658-1664.

UCEV-Base de datos (Unió de Cooperatives d'Ensenyament Valencianes) (2016) Mapa de centros. http://www.ucev.coop/mapa-de-centres/ [Consultada: 17/06/16].

UCEV (2016) VII Declaració Educativa de la Unió de Cooperaties d'Ensenyament Valencianes. http://www.ucev.coop/declaracio-educativa/.

Wu, S., Straub, D.W. y Liang, T. (2015) How information technology governance mechanisms and strategic alignment influence organizational performance: Insights from a matched survey of business and IT managers. Mis Quarterly, $\mathrm{N}^{\circ}$ 39, Vol. 2, pp. 497-518. 